\title{
Non-Axisymmetric Structure in the Satellite Dwarf Galaxy NGC2976: Implications for its Dark/Bright Mass Distribution and Evolution
}

\author{
Octavio Valenzuela ${ }^{1}$, Hector Hernandez-Toledo ${ }^{1}$, Mariana Cano-Díaz ${ }^{1,2}$, Ivanio Puerari ${ }^{3}$, \\ Ronald Buta ${ }^{4}$, Bárbara Pichardo ${ }^{1}$, Robert Groess ${ }^{5}$ \\ ${ }^{1}$ Instituto de Astronomía,Universidad Nacional Autonóma de Mexico, A.P. 70-264, 04510, México, \\ D.F.,octavio@astro.unam.mx \\ ${ }^{2}$ Osservatorio Astronomico di Roma - INAF. Via di Frascati 33, 00040 Monte Porzio Catone, Italy \\ ${ }^{3}$ Instituto Nacional de Astrofísica Optica y Electrónica. Calle Luis Enrique Erro 1, 72840, Sta. Maria \\ Tonantzintla, Puebla, Mexico \\ ${ }^{4}$ Department of Physics and Astronomy, University of Alabama, Tuscaloosa, AL 35487, USA. \\ ${ }^{5}$ School of Computational and Applied Mathematics, University of Witwatersrand, Private Bag 3, WITS \\ 2050, South Africa
}

\begin{abstract}
We present the result of an extensive search for non-axisymmetric structures in the dwarf satellite galaxy of M81: NGC 2976, using multiwavelength archival observations. The galaxy is known to present kinematic evidence for a bysimmetric distortion (Spekkens \& Sellwood 2007; Sellwood \& Sánchez 2010; Adams et al. 2011) however the stellar bar presence is controversial. This controversy motivated the possible interpretation of NGC 2976 presenting an elliptical disk triggered by a prolate dark matter halo (Simon et al. 2003; Kazantzidis et al. 2010a). We applied diagnostics used in spiral galaxies in order to detect stellar bars or spiral arms. The $\mathrm{m}=2$ fourier phase has a jump around 60 arcsecs consistent with a central bar and bisymmetric arms. The $\mathrm{CO}, 3.6 \mu$ surface brightness and the dust lanes are consistent with a gas rich central bar and possibly with gaseous spiral arms. The barlike feature is close to 20 degrees offset the disc position angle, in agreement with kinematic estimations. The kinematic jumps related with the dust lanes suggest that the bar perturbation in the disk kinematics is non-negligible and the reported noncircular motions, the central gas excess and the nuclear X-ray source (AGN/Starburst) might be produced by the central bar. SPH simulations of disks inside triaxial dark halos suggest that the two symmetric spots at 130 arcsecs and the narrow arms may be produced by gas at turning points in an elliptical disk, alternatively the potential ellipticity can be originated by tidally induced strong stellar bar/arms, in both cases rotation curve interpretation is importantly biased. The M81 group is a natural candidate to trigger the bisymmetric distortion and the related evolution as suggested by the HI tidal bridge detected by Chynoweth et al. (2008). We conclude that both mechanisms, the gas rich bar and spiral arms triggered by environment (tidal stirring), and primordial halo triaxiality, can explain most of NGC 2976 non-circular motions, mass redistribution and nuclear activity. Distinguishing between them requires detailed modeling of environmental effects. A similar analysis like ours may reveal such kind of structures in other nearby dwarf satellite galaxies, if this is confirmed, the same evolutionary scenario will be applicable to them. This implies biases constraining their dark matter distribution and also making comparison against theoretical predictions for isolated galaxies.
\end{abstract}




\section{Introduction}

The internal kinematics of galaxies has been one of the central elements that lead to initially establish the dark matter hypothesis in astrophysics (Rubin et al. 1978; Bosma 1978). In particular the non-keplerian behavior of disk galaxies rotation curves has been naturally incorporated into the current cosmological paradigm, the LCDM model, through dark matter halos surrounding galaxies, and even the halo mass assembly is accurately predicted by the model (Frenk et al. 1988, e.g.). The assumption of a nearly full rotational support of disk galaxies is critical in order to compare cosmological predictions with data. Taking this comparison strategy further in detail it has been found that LSB/dwarf galaxies may reveal a potential flaw for the most simple cosmological predictions for rotation curve shapes that neglect baryonic physics, the so called core-cusp problem (Moore 1994; Flores \& Primack 1994; de Blok 2010) . Including the effect of baryons although challenging, offers possible solutions like modifying the internal halo structure (Mashchenko et al. 2006; Governato et al. 2010) or biasing the interpretation of kinematics because of galaxy structure or pressure support (Rhee et al. 2004; Valenzuela et al. 2007). The possibility of distinguishing between different galaxy formation scenarios makes important and interesting to quantitatively evaluate possible deviations from the rotational support assumption, the so called noncircular motions. They have been largely discussed either motivated by the observed structure of some galaxies like bars, spirals, lopsidedness (Rhee et al. 2004; Spekkens \& Sellwood 2007; Sellwood \& Sánchez 2010) or the possibility of elliptical galaxy disks triggered by triaxial dark matter halos but masked by projection effects (Hayashi \& Navarro 2006). The pursue for adopting methods free from the non-circular motions complications has triggered a revision of both theoretical (Bailin et al. 2007) and observational analysis techniques (Kuzio de Narav et al. 2012; Kuzio de Naray \& Kaufmann 2011; Spekkens \& Sellwood 2007). Currently in observations, the presence of non-circular motions are a common result, besides almost every new study reports galaxies whose rotation curves are consistent with cores but also a minority consistent with cusps (Simon et al. 2005), in some cases cuspier than cosmological predictions, suggesting some systematic effect. However, still there is not an unanimous agreement about the interpretation of these observational results, in particular for the detected non-circular motions, for example some valuable methods assume the epicyclic approximation which may be problematic for strong perturbation like bars or considerably elliptical disks (Franx et al. 1994; Schoenmakers et al. 1997), others do not adopt these hipothesis (Spekkens \& Sellwood 2007; Sellwood \& Sánchez 2010; Kuzio de Naray et al. 2012). Some other methods assume a subdominant and random nature to non-circular motions (Oh et al, 2011).

Recently, it has been shown that bar instabilities are present in some dwarf LSB galaxies used to study the central dark matter distribution using their rotation curve (NGC 3109, NGC 6822 Valenzuela et al. (2007)). The bar dynamical effects are arguably enough to explain the discrepancy with cosmological predictions, at least when they act together with pressure support (Valenzuela et al. 2007). For one dwarf galaxy (NGC 6822) the bar has been clearly revealed in HI kinematics residuals after rotation substraction (see figure 2 in Rhee (2009), Rhee et al . in preparation) questioning if the rotation curve fully constrains the mass distribution for this galaxy. It is however necessary to analyze more dwarf galaxy cases cases before arguing that this is a general result. On the contrary, NGC 2976 belongs to a sample of galaxies that has been argued to present a pure disk, with no bar, spiral arms or bulge, the clear detection of non-circular motions raised-up the possibility of being witnessing an elliptical disk triggered by the dark halo triaxiality (Simon et al. 2003, 2005). Lately, Spekkens \& Sellwood (2007) reported evidence favoring a kinematic bisymmetric distortion over an inflow/outflow, leaving open the question if the kinematic perturber is a stellar bar or a prolate dark matter halo. More recently Adams et al. (2011) found stellar kinematic evidence for non-circular motions possibly consistent with a disk inside a cuspy halo. Although recent analysis of 2MASS images of NGC 2976 (Menéndez-Delmestre et al. 2007) marginaly suggested the presence of a stellar bar candidate, more recent theoretical studies conclude that based on the photometrically estimated disk mass, the triaxiality must have been preserved after the 

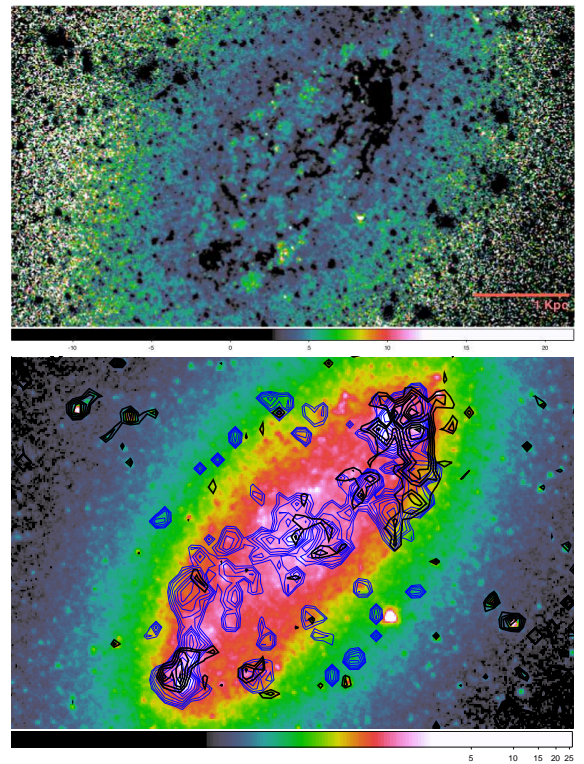

Fig. 1.- Morphological Bar Evidence. Upper panel: Color Map B-3.5 $\mu$, the curved dust lanes in the central region are characteristic of a weak/slow bar. The upper right region suggest the geometry of spiral arms. Lower panel shows the $3.6 \mu$ image tracking the stellar component with few dust extinction, combined with dust map contours (thick black) and CO intensity contours (blue thin). The central region presents a box like structure resembling a weak stellar bar and is spatially coincident with the curved dust lanes. The contours for integrated intensity $\mathrm{CO} \mathrm{J}=0->1, \mathrm{CO} \mathrm{J}=2->1$, along each line of sight considering regions with $I_{C O}>2 \sigma$ (taken from Bolatto et al. in peparation and Leroy et al. (2009)). Notice the change in position angle and the coincidence with boxy stellar structure as well with the dust lanes.

disk formation (Kazantzidis et al. 2010a), making appealing the triaxial halo presence scenario. It is then important to quantitatively asses the bar presence/absence, together with measurements of its strength. A possible bar may represent evidence of secular evolution in dwarf irregular galaxies, which importance is currently uncertain in evolution models. In this paper we used techniques commonly used in giant high surface brightness galaxies, in order to confirm the bar presence and also with the aim to measure the strength of the perturbation with enough signal to noise. The paper is structured as follows: Section 2 describes

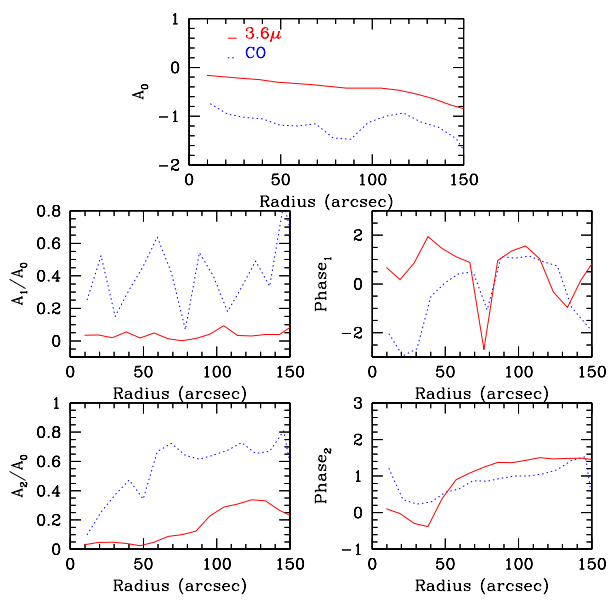

Fig. 2.- Azimuthally averaged 1st and 2nd fourier modes. Top panel: Mean surface brightness profile in stellar component (solid) and molecular gas CO (dotted). Medium left: A1 fourier mode (lopsidedness). Medium right: A1 phase profile in radians. Lower left: A2 fourier mode (bisymmetric distorsion) amplitude. Notice the A2 mode amplitude in $\mathrm{CO}$ is large while in $3.6 \mu$ is negligible, suggesting the bar gaseous richness. The A2 mode phase shows a big jump close to 50 $\operatorname{arcsec}(0.5 \mathrm{kpc})$, coincident with the begining of spiral arms

the observations used in this study. Section 3 describes the different diagnostics performed in NGC 2976 in order to detect and measure the bar/arm properties. Section 4.1 presents a discussion about the nature of non-circular motions and their relationship with a bar or non spherical halo. Section 4.2 briefly discusses the consequences for the detected bar and arms for satellite galaxies transformation models in groups like tidal stirring. Finally in section [5 we present our conclusions.

\section{Observations}

Daigle et al. (2006) describes NGC 2976 as a peculiar dwarf $\left(M_{B}=16.90\right)$ late-type (SAc pec) galaxy with a nearly linear rotation curve, no spiral arm visible and two strong HII regions located on each side of the galaxy. They report a Photometic/Kinematical P.A. and Inclinatios of $323 / 323.5$ and $63 / 70$ respectively. Stil \& Israel (2002) observed that in HI the rotation curve seems to flatten near the edge of the HI disc. Ac- 
cording to Bronkalla, Notni \& Mutter (1992), the outer parts of NGC 2976 have been undisturbed for a long time and are very old (5 Gy, probably up to 15 Gy). This last conclusion seems to be at odds with the recent results in Walter et al. 2002 and Chynoweth et al. 2008 who observed the M81/M82 group finding that M81, M82, NGC 3077, and NGC 2976 show the remnants of strong interactions as well as over 40 dwarf galaxies in their close neighborhood. Chynoweth et al. 2008 studied the HI emission of the group covering an area $3^{\text {deg }} \times 3^{\text {deg }}$ centered on M81 to include NGC 2976 and the extended emission associated the group finding an $\mathrm{HI}$ cloud located $27 \mathrm{kpc}$ to the northeast of NGC 2976 and calculating a mass of $2.67 \pm 0.65 \times 10^{7} M_{\odot}$ which may be contributing to the observed perturbation in NGC 2976.

NGC 2976 is part of the Spitzer Infrared Nearby Galaxies Survey (SINGS) sample and our photometric analysis take advantage of the already available $3.6 \mu \mathrm{m}$ IRAC images with a scale of 1.22 $\operatorname{arcsec} /$ pix. We combined these data with observations at visible wavelengths, mainly the $B_{J}$ band images of the photometric system by Gullixson et al. (1995) as observed by Frei et al. (1996) and scale of $1.35 \mathrm{arcsec} / \mathrm{pix}$. We also use $\mathrm{H} \alpha$ monochromatic maps from the FabryPerot of New Technology for the Observatoire du mont Megantic (FaNTOmM) with scale of $1.61 \mathrm{arcsec} / \mathrm{pix}$ and described in (Daigle et al. 2006). After matching the images to the same resolution, we built a $(B-3.6)$ color index map to get new insights on the structural properties of NGC 2976. For some of the analysis we tried to deproject the image assuming a circular disk and a disk thickness consistent with galaxies with similar rotation curve amplitude, we avoided the deprojection in some cases because the possible non-axisymmetric structure and inclination may complicate the traditional strategy (García-Gómez et al. 2004).

\section{Diagnostics for the Bar Presence}

\subsection{Morphological Evidence: Dust Lanes, CO Intensity maps}

It is well established in galaxy dynamics that a galaxy bar produces interstellar medium schocks wich can be traced by dust lanes Athanassoula 1992; Prendergast 1983). In order to seek for the presence of schocks we constructed a B-3.6 $\mu$ color image presented in the upper panel of figure 1 The dark structures are the most dust obscured regions. Particularly notable are the pair of curved dust lanes in the central region of NGC 2976, similar to the ones pesented by Athanassoula (1992) in the case of weak or slow bars. Near to the color map image upper right spot there is a spiral arm like dust structure. This finding supports the existence of a disk bar and spiral arms, however it can still be argued that a random star formation pattern may originate the structures, therefore we will study other diagnostics like the kinematic response traced by $\mathrm{H}-\alpha$ in the bar region in the following sections. As a support to the bar interpretation we present at the lower panel of figure 1 the $\mathrm{CO}$ integrated intensity contours in NGC 2976, taken from the HERACLES survey (Leroy et al. 2009) and the STING survey (Bollato et al in preparation) on top of the $3.6 \mu$ image, and together with the color map contours. An elongated CO structure with different position angle compared with the average stellar component is outstanding, the structure twists on both sides developing narrow arcs similar to tightly wounded spiral arms. The spiral arms like features are coincident with the dust map structures. The two symmetric bright spots suggest the beginning of spiral arms.

\subsection{Radial Fourier modes}

A bisymmetric structure like a galactic bar or a spiral arm pair can be well represented by even fourier components. A first diagnostic to the presence of such structures is the amplitude of the second fourier mode and the behaviour of its phase. Figure 2 shows the first two fourier modes for the average stellar component and the $\mathrm{CO}$ gas emission (solid and dashed line respectively). The first fourier mode shows a very small amplitude in the stellar component (less than 0.05), while for the $\mathrm{CO}$ tracer the behaviour is noisier but with a larger amplitude. The phase is consistent for both tracers. It is not straight forward to disentangle if this amplitude is the result of the clumpy distribution of molecular gas or a truly global lopsidedness. The second fourier mode barely has a non zero amplitude inside 70 arcsecs in $3.6 \mu$ where the isophotes show a boxy shape, however its amplitude in CO indicates that $20 \%$ of the surface brightness is in the $m=2$ mode, consistent to the more elongated $\mathrm{CO}$ distribution. The correspond- 


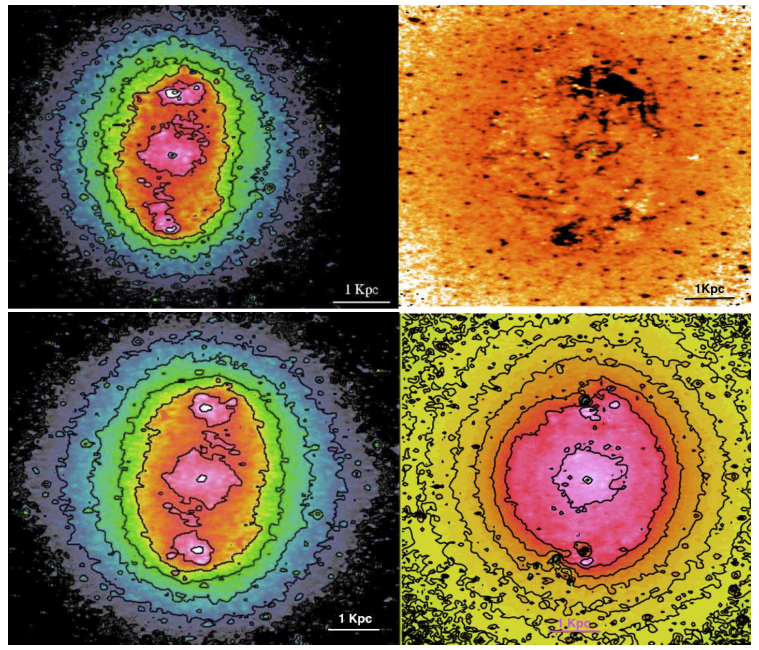

Fig. 3.- Even and odd structure. The figure shows 4 images. Upper left panel presents the original Spitzer image in 3.6 microns, deprojected under the assumption of a circular external disk. Upper right hand side shows a deprojected B3.6 color map. The lower left presents an image constructed only with the even powers of a 2-dimensional fourier decomposition. Notice the spiral/oval structure. The Lower right hand image, shows am image constructed with only odd powers. The circular yellow contours suggest the the galaxy disk is not lopsided in the external region.

ing phase is consistent in both tracers, showing a big jump at 50-70 arcsecs, suggesting the transition from the bar to the spiral arms. Azimuthal average and projection effects may have a nontrivial influence on one dimensional fourier analysis, not to mention the flocculent structure, therefore we will apply other diagnostics.

\subsection{Two Dimensional Fourier Analysis}

In order to keep azimuthal information we performed a 2-Dimensional fourier analysis of the 3.6 $\mu$ image. We splitted the image in two: one image built with all the even modes and another one built with the odd modes. If there is a bar or bisymmetric arms it must be shown up in the even modes image. Figure 3 shows at the top the deprojected $3.6 \mu$ image next to a de-projection of the color map and at the bottom both even and odd images (left and right respectively). The even im-
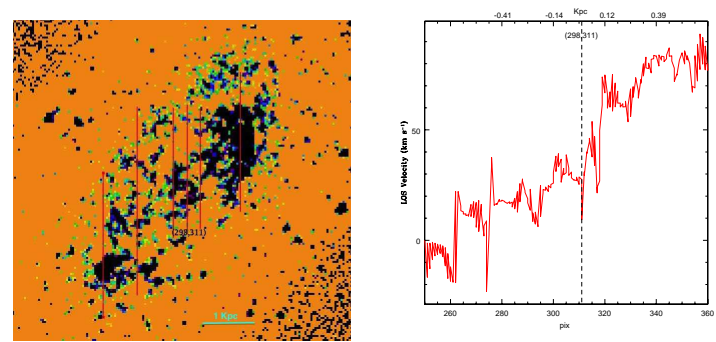

Fig. 4.- Dynamical Effect of the Bar. Left panel presents the color map showing dust lanes across the whole galaxy. The vertical lines show cuts along the velocity field crossing the dust lanes. Specifically the vertical lane labeld 298, 311 crosses the curved dust lane at this pixel coordinates. The right panel shows how the velocity profile presents a jump of about $30 \mathrm{~km} / \mathrm{s}$ going from pixel 330 to 311, in other words crossing the dust lane.

age clearly shows a boxy structure at the position of the CO bar and the beginning of bi-symmetric spiral arms. Although encouraging complications associated with the de-projection have to be carefully handled (García-Gómez et al. 2004), it is however remarkable that bar and spiral arm structures are so well defined despite the radial Fourier mode in the stellar component is not large, supporting the interpretation that both bar and arms are gas rich but they have a stellar counterpart.

\subsection{Kinematic Signatures of Bar Induced Shocks}

Shocks across dust lanes are readily identified as steep velocity gradients Athanassoula 1992; Weiner et al. 2001). We used the $\mathrm{H}-\alpha$ velocity field kindly previously discussed by Daigle et al. (2006). The data points in the right panel of figures 4 show the observed velocities along a pseudoslit crossing the velocity field placed with an orientation perpendicular to the curved dustlanes as shown in its left panel. The pseudo-slit reveals a projected velocity gradient of almost $30 \mathrm{~km} \mathrm{~s} 1$ is coincident with the prominent dust lane. A similar configuration and signature is discussed in (Zánmar Sánchez et al. 2008). We conclude that regardless of projection effects, the gas-rich bar has a dynamical effect on NGC2976 internal kinematics. 


\subsection{Two-Dimensional Force Map}

Once we are confident about the presence of a bar in the stellar component of NGC 2976, it is natural to ask for the bisymmetric perturbation strength. Buta \& Block (2001) introduced the ratio of tangential to radial force $Q_{b}$ parameter as a diagnostic of the bar/arm strength. After solving the poisson equation on the deprojected stellar component image, the potential is diferentiated in order to calculate radial and tangential forces point by point. Afterwards we built a two dimensional map of the ratio as is shown is figure [5] The characteristic bar/arms signature is the alternating sign of this ratio as we move through quadrants, verifying the bar/arms interpretation in our previous diagnostics. The central region shows a slightly asymmetric central pattern pattern and the large scale diagram ilustrate the spiral arms force as is discussed by Block et al. (2004) The ratio of the maximum tangential force to the average radial axisymmetric amplitude has been shown as an amplitude measurement Combes \& Sanders 1981), for NGC 2976 the value in the bar region is around 0.4 , therefore regardless of its nature the bi-symmetric perturbation is a strong one. At the arms region the tangential perturbation is even larger reaching values of $80 \%$ of the radial force.

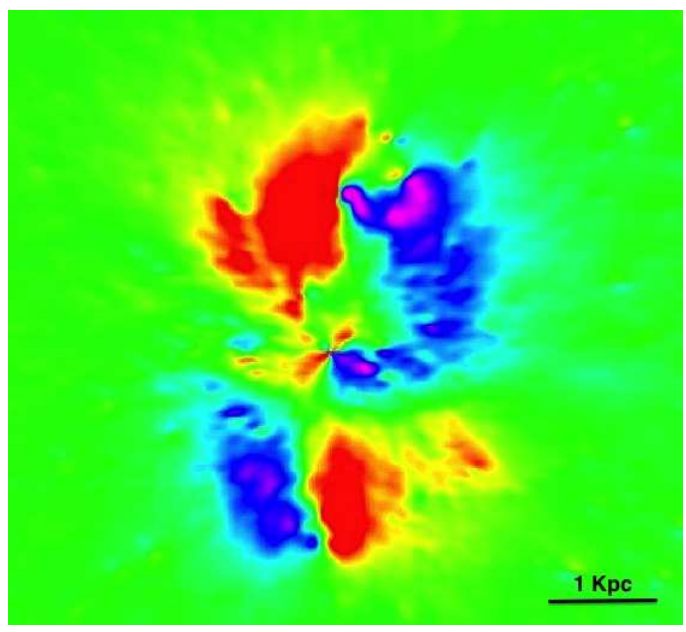

Fig. 5. - .Torque Map based on the $3.6 \mu$ image and solving the Poisson equation. Red (Blue) colors correspond to negative (positive sign). The alternating sign is the distinctive signature of a bar/arm quadrupole.

\section{Dark Matter Distribution}

NGC 2976 dark mater distribution has been discussed by several studies, most of them favored a cored halo distribution based on interpretation of the rotation curve (Simon et al. 2003; Spekkens \& Sellwood 2007; de Blok et al. 2008). However, recently Adams et al. (2011) concluded that stellar kinematics and anisotropic Jeans modeling combined with information of its star formation history favored a cuspy dark matter distribution. A critical difference between these studies is the interpretation of non-circular motions. These non-circular motions are detected both in gas and stellar component. In the next sections we will discuss their possible nature.

\subsection{Non Circular Motions: Triaxial Halo/ Bar-Arms?}

NGC 2976 has been proposed as a system that provides kinematic evidence for a triaxial halo. This is important because the existence of nonspherical dark matter halos is an ubiquitous prediction of hierarchical structure formation model. Galaxy disks are expected to react to the triaxial halo potential become elliptical and even develop instabilities triggered by the halo perturbation, the halo triaxiality is also modified by the process, making non-trivial the predicitons about the central density structure (Bailin et al. 2007). However, a positive detection of halo triaxiality in disk galaxies has been hampered partly because of the degeneracy between true disk ellipticity and projection effects. A promising avenue to verify this prediction is a kinematic detection of disk ellipticity in the absence of bars and spiral arms. Simon et al. (2003) pointed out that NGC2976 shows considerable non-circular motions but the galaxy lacks of an obvious non-axisymetrics internal baryonic perturber, raising the possibility that we are witnessing an elliptical disc triggered by a triaxial halo. Spekkens \& Sellwood 2003 showed that a bisymmetric model is preferred by the data over a radial flow. Furthermore, recently Kazantzidis et al 2010 showed that constraints on the disk mass suggest that adiabatic disk formation would have not been able to erase halo triaxiality. Although our results do not question the theoretical calculations, we positively detect a bar and spiral arms. It is fair to mention that the 
bar and arms system is unusual, the bar is clearly shown in $\mathrm{CO}$ intensity map, but in $\mathrm{HI}$ the bar is rather uncertain but arms are detectable. In $3.6 \mu$ the bar hint is only a boxy like structure, and the arms are noticeable mainly through the symmetric bright spots. The situation in normal galaxies is the opposite, a clear stellar bar is observed and some gaseous response to the dynamical perturbation hinting the bar. If NGC 2976 gaseous disks is dominant over stars is possible to explain its morphology. However, the reported stellar mass is $5 \times 10^{8} \mathrm{M}_{\odot}$ and the gaseous mass at most $30 \%$ of stars $\left(1.5 \times 10^{8} \mathrm{M}_{\odot}\right)($ Del Popolo 2012). Although there is evidence of gas tidal stripping therefore the galaxy may have been even richer in gas, there is not obvious reason of why the bar should be more visible in gas. Williams et al. (2010) and others have discussed the presence of a stellar spheroid or halo of intermedia age stars, this structure may be observed in projection as a boxy bulge. One possible alternative interpretation is that indeed we are witnessing the gas/stars response to an elongated dark matter halo, in such a case stars reacted to the triaxial halo orbital structure developing the boxy bulge. Indeed, disk non-axisymmetric structures and triaxiality are not necessarily mutually exclusive. It is well known that an elongated halo is able to trigger spiral arms (Bekki \& Freeman 2002) Some recent studies simulated the interaction of bars and disks inside triaxial live halos, concluding that disk bar formation is possible but it can also erase halo triaxiality in the central region (Berentzen et al 2005, Athanassoula et al 2010). However the models were tuned to high surface brightness galaxies unlike NGC 2976, therefore because of its low baryonic fraction, we can not rule out the possibility that considerable triaxiality has survived in the central region of NGC 2976 regardless of the bar/arms presence. We conclude that non-circular motions in the central 80 arcsecs are likely triggered by the detected bar/arms, and is at least contrived to compare the importance of bar/arms against halo triaixiality inside 50 arcsecs. Nevertheless, triaxiality may survive at the galaxy outskirts. In order to test this interpretation we used controlled SPH simulations of gaseous disks in triaxial halo potentials. The simulations will be described in detail somewhere else (Pichardo et al in preparation), however here we present only the basic information. The halo has a logarithmic potential with a very small core radius, mimicking a cusp-like halo model. A Kuzmin gaseous disk with an isothermal equation of state is initially set in equilibrium inside an spherical halo, later during 20 dynamical times at twice the initial disk radius (adiabatically), the halo model axis ratios are gradually modified, recently a similar study was described in the literature (Khoperskov et al. 2012). The disk responds developing ellipticity, and spiral arms, and the gas inflow triggers a bar like central structure which realism is uncertain because the idealized simulation set up. An interesting finding is that for disk plane ellipticity of 0.3-0.4 or larger the gas at turn around piles up and in the more eccentric cases develops two symmetric shocks. NGC 2976 shows two symmetric bright spots in the stellar photometry and $\mathrm{H}$ alpha, suggesting that either the spiral arms are stronger than expected or the disk ellipticity at that radius is near $0.3-0.4$ or greater.
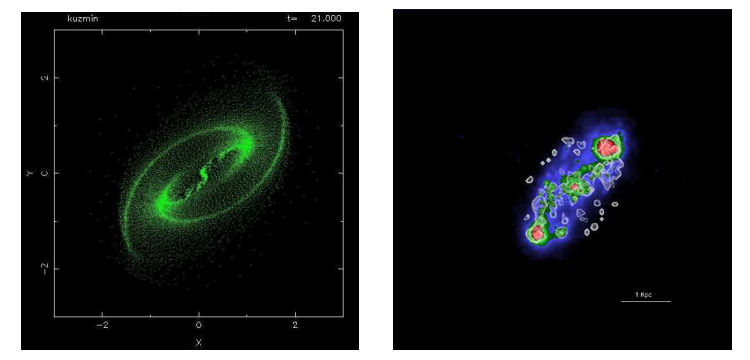

Fig. 6.- Symmetric Bright Spots. First panel: SPH gas simulation of a Kuzmin disk inside a triaxial Dark Matter halo. The disk was introduced adiabatically, after some dynamical times, spiral arms are developed and gas at turning points piles up along the line of nodes creating an over-density and either a shock for ellipticities larger than 0.5 . Notice the symmetric overdense regions where presumably star formation may happen. Second panel: $24 \mu$ Spitzer/MIPS image tracking dust warmed by star formation. Noticed the two symmetric bright star formation regions. They could be evidence of gas turn around triggered by a prolate dark matter halo as in the simulation, or alternative by strong bar/spiral arms. 


\subsection{Environment Triggered Evolution of Satellite Dwarf Galaxies}

Once we have confirmed the presence of a gas rich non-axisymetric structure in the disk of NGC 2976, the most natural explanation is that the tidal interaction with the environment triggered a mass redistribution, making the galaxy unstable regardless of the low baryonic mass fraction. Specifically, N2976 environment is the M81 group, an extended HI tidal tail suggests that the galaxy has indeed suffered an interaction with the M81M82 system. The whole M81 group mass is close to $10^{12} \mathrm{M}_{\odot}$ (Karachentsev et al. 2006) and the projected distance up to NGC 2976 is close to $190 \mathrm{kpc}$, therefore a crude estimation for the crossing time is 1 gigayear. Chynoweth et al. (2008) estimates 0.2-0.3 gyrs for the age of the neutral gas bridge connecting M81-M82 which based on the bridge projected extension, is younger than the one connecting M81 and NGC 2976, therefore a 1 gigayear estimate for their interaction age is conservative and also in agreement with (Williams et al. 2010) In comparison, at the last measured optical rotation curve point $(2 \mathrm{kpc})$ the NGC 2976 rotation amplitude is $80 \mathrm{~km} / \mathrm{s}$, therefore the rotation period is approximately 0.024 gigayears, suggesting that enough time has spent since the interaction in such a way NGC 2976 disk should be relaxed by now. We conclude that disk self-gravity is important in NGC 2976 and as a consequence the bar and spiral arms are unlikely transient structures. Recently a low luminosity AGN has been revealed in NGC 2976 through X-Ray and NIR observations (Grier et al. 2011), this probably reveals the presence of gas inflows triggered by the non-axisymmetric structure and also of energy injection into the central galaxy ISM , both processes may have effect on the dark matter halo structure.

\subsection{Generality}

A critical question to ask is: How common is NGC2976 between satellite dwarf galaxies? The are two ways to address such an inquire: through statistical analysis of satellite galaxy population or using theoretical predictions. There are not many systematic studies searching for bars in satellite galaxies around normal galaxies. Statistical analysis of more massive galaxy groups show that
AGNs potentially triggered by secular evolution are more common in satellites than in central galaxies (Allevato et al. 2012), however there are not many similar studies in less massive groups like M81. It is natural to ask if the whole evolution including disk instability and nuclear activity is triggered by the environment and if a similar situation may be common to other satellite dwarf galaxies located in low density groups like M81 or the Local Group. Recently (Grier et al. 2011) found AGN candidates in a sample of THINGS galaxies where NGC 2976 is included, however more work is required. From the theoretical side, the scenario is a low red-shift incarnation of the so called tidal stirring one (Mayer et al 2001). Kazantzidis et al. (2010b) recently characterized the evolutionary tracks of satellite dwarf galaxies finding that bar instabilities developed before a global mass redistribution happened, the evolutionary stage seems quite insensitive to orbit details and more dependent of the cumulative tidal force(Lokas et al. 2010), then is more sensitive to the satellite accretion time. However, more recent calculations including SN feedback of gas rich dwarf galaxies, seem to show that no bar instabilities developed in galaxies similar to NGC 2976, likely because of gas inflow may inhibit bar grow (Mayer 2011). Finding a bar and spiral arms in NGC2976 is a good constraint to this model. Based on NGC2976 we can especulate that the energy injection triggered by the low luminosity AGN/nuclear starburst may constraint the gas inflow allowing the bar growth. Further insight into this matter may be obtained analyzing more satellite galaxies in M81 and other nearby low density groups (Hernandez-Toledo, Valenzuela et al in preparation), as a matter of fact some hints suggesting environmental transformation of satellite galaxies in low mass groups may be already reported in M101 group (Mihos et al. 2012). Another consequence of this scenario is that the transformation process triggered by environment may bias comparison against theoretical predictions for isolated galaxies, including expected mass-Vmax-concentration relationships, triaxiality, star formation rate (Colín et al. 2010; Avila-Reese et al. 2011; Rodríguez-Puebla et al. 2012) and how these properties correlate to the baryonic structure of the galaxies residing inside dark matter subhalos. 


\section{Conclusions}

Four different indicators: CO intensity map morphology, curved central dust lanes, fourier $\mathrm{m}=2$ mode surface brightness decomposition in 1$\mathrm{D}$ and $2-\mathrm{D}$ and $\mathrm{H}-\alpha$ kinematic jumps across the central dust lanes, all suggest that the NGC 2976 disk shows a non-axisymetric structure: a gas rich central bar structure and gaseous spiral arms at large scales.

The gas rich bar/arms is expected to produce non-circular motions biasing constraints on the mass distribution estimation in agreement with recent kinematic analysis of NGC 2976 (Adams et al. 2011), similar structures may bias comparison of this galaxy versus cosmological predictions for isolated galaxies (Rodríguez-Puebla et al. 2012)

The bar and arms are the most natural triggers of the observed non-circular motions, mass redistribution and nuclear activity $(\mathrm{AGN} /$ nuclear starburst, Grier et al. (2011) ), however a detailed modeling of the galaxy and its environment is required in order to decide between environment induced bar/arms and triaxial dark matter halo induced elliptical disk and arms.

If the bar/arms are not as strong as CO observations suggest, the two symmetric spots visible in $\mathrm{H}-\alpha$, and infrared, are possibly indicating an elliptical disk with ellipticity greater than 0.3 .

The bar existence is consistent with tidal stirring predictions, the recently reported low luminosity AGN/nuclear starburst may be connected with the bar and may be a common stage on satellite galaxies evolution, with possible influence in the star formation history.

Overall, NGC 2976 considered inside a sample of simple, isolated, axisymmetric dwarf galaxies, is revealed as a complex galaxy affected by its environment and showing peculiar spiral arms and bar. Secular induced mass redistribution is likely important and connected with the reported AGN/nuclear starburst activity, the same conclusions likely apply to the evolution of many other dwarf satellite galaxies.

\section{Acknowledgments}

We thank P. Amram for kindly making available the fabry-perot data used in this paper. $\mathrm{H}$.
Segura is acknowledged for help with graphic material. We thank valuable conversations with: G. Rhee, J. Simon, J, Navarro, P. Teuben, A. Bosma, K. Spekkens. Support by the UNAM PAPIIT IN118108 grant is acknowledged. OV acknowledges support from grant PAPIIT/UNAM: IN112313. MCD was supported by the Marie Curie Training Network ELIXIR under the contract PITN-GA-2008-214227 from European Commission.

\section{REFERENCES}

Adams, J. J., Gebhardt, K., Blanc, G. A., et al. 2011, arXiv:1110.5951

Allevato, V., Finoguenov, A., Hasinger, G., et al. 2012, ApJ, 758, 47

Athanassoula, E. 1992, MNRAS, 259, 345

Avila-Reese, V., Colín, P., González-Samaniego, A., et al. 2011, ApJ, 736, 134

Bailin, J., Simon, J. D., Bolatto, A. D., Gibson, B. K., \& Power, C. 2007, ApJ, 667, 191

Bekki, K., \& Freeman, K. C. 2002, ApJ, 574, L21

Block, D. L., Buta, R., Knapen, J. H., et al. 2004, AJ, 128,183

Bosma, A. 1978, Ph.D. Thesis,

Buta, R., \& Block, D. L. 2001, ApJ, 550, 243

Buta, R., Block, D. L., \& Knapen, J. H. 2003, AJ, 126,1148

Chynoweth, K. M., Langston, G. I., Yun, M. S., Lockman, F. J., Rubin, K. H. R., \& Scoles, S. A. 2008, AJ, 135, 1983

Combes, F., \& Sanders, R. H. 1981, A\&A, 96, 164

Colín, P., Avila-Reese, V., Vázquez-Semadeni, E., Valenzuela, O., \& Ceverino, D. 2010, ApJ, 713, 535

Daigle, O., Carignan, C., Amram, P., Hernandez, O., Chemin, L., Balkowski, C., \& Kennicutt, R. 2006, MNRAS, 367, 469

de Blok, W. J. G., Walter, F., Brinks, E., et al. 2008, AJ, 136, 2648 
de Blok, W. J. G. 2010, Advances in Astronomy, 2010 ,

Del Popolo, A. 2012, MNRAS, 419, 971

Franx, M., van Gorkom, J. H., \& de Zeeuw, T. 1994, ApJ, 436, 642

Flores, R. A., \& Primack, J. R. 1994, ApJ, 427, L1

Frenk, C. S., White, S. D. M., Davis, M., \& Efstathiou, G. 1988, ApJ, 327, 507

Frei, Z., Guhathakurta, P., Gunn, J. E., \& Tyson, J. A. 1996, AJ, 111, 174

García-Gómez, C., Barberà, C., Athanassoula, E., Bosma, A., \& Whyte, L. 2004, A\&A, 421, 595

Grier, C. J., Mathur, S., Ghosh, H., \& Ferrarese, L. 2011, ApJ, 731, 60

Governato, F., et al. 2010, Nature, 463, 203

Hayashi, E., \& Navarro, J. F. 2006, MNRAS, 373, 1117

Hernández-López, I., Athanassoula, E., Mújica, R., \& Bosma, A. 2009, Revista Mexicana de Astronomia y Astrofisica Conference Series, 37, 160

Holmberg, E. 1974, Arkiv for Astronomi, 5, 305

Iono, D., Yun, M. S., \& Mihos, J. C. 2004, ApJ, 616, 199

Kazantzidis, S., Abadi, M. G., \& Navarro, J. F. 2010, ApJ, 720, L62

Kazantzidis, S., Lokas, E. L., Callegari, S., Mayer, L., \& Moustakas, L. A. 2010, arXiv:1009.2499

Klypin, A.A., Kravtsov, A.V., Valenzuela, O., \& Prada, F. 1999, ApJ, 522, 82

Klypin, A., Gottlöber, S., Kravtsov, A. V., \& Khokhlov, A. M. 1999, ApJ, 516, 530

Khoperskov, A. V., Eremin, M. A., Khoperskov, S. A., Butenko, M. A., \& Morozov, A. G. 2012, Astronomy Reports, 56, 16

Kuzio de Naray, R., Arsenault, C. A., Spekkens, K., et al. 2012, arXiv:1209.4653
Kuzio de Naray, R., \& Kaufmann, T. 2011, MNRAS, 414, 3617

Leroy, A. K., Walter, F., Bigiel, F., et al. 2009, AJ, 137, 4670

Lokas, E. L., Kazantzidis, S., Mayer, L., \& Callegari, S. 2010, arXiv:1011.3357

Mashchenko, S., Couchman, H. M. P., \& Wadsley, J. 2006, Nature, 442, 539

Mayer, L. 2011, EAS Publications Series, 48, 369

Menéndez-Delmestre, K., Sheth, K., Schinnerer, E., Jarrett, T. H., \& Scoville, N. Z. 2007, ApJ, 657,790

Mihos, C., Keating, K., Holley-Bockelmann, K., Pisano, D. J., \& Kassim, N. 2012, arXiv:1210.8333

Moore, B. 1994, Nature, 370, 629

Navarro, J. F., Abadi, M. G., \& Steinmetz, M. 2004, ApJ, 613, L41

Prendergast, K. H. 1983, Internal Kinematics and Dynamics of Galaxies, 100, 215

Rhee, G., Valenzuela, O., Klypin, A., Holtzman, J., \& Moorthy, B. 2004, ApJ, 617, 1059

Rhee, G. 2009, Galaxy Evolution: Emerging Insights and Future Challenges, 419, 53

Rahman, N., Bolatto, A. D., Xue, R., et al. 2012, ApJ, 745, 183

Rodríguez-Puebla, A., Drory, N., \& Avila-Reese, V. 2012, ApJ, 756, 2

Roychowdhury, S., Chengalur, J. N., Karachentsev, I. D., \& Kaisina, E. I. 2013, arXiv: 1308.6200

Rubin, V. C., Thonnard, N., \& Ford, W. K., Jr. 1978, ApJ, 225, L107

Sales, L., \& Lambas, D. G. 2004, MNRAS, 348, 1236

Sellwood, J. A., \& Sánchez, R. Z. 2010, MNRAS, 404, 1733

Simon, J. D., Bolatto, A. D., Leroy, A., \& Blitz, L. 2003, ApJ, 596, 957 
Simon, J. D., Bolatto, A. D., Leroy, A., Blitz, L., \& Gates, E. L. 2005, ApJ, 621, 757

Schoenmakers, R. H. M., Franx, M., \& de Zeeuw, P. T. 1997, MNRAS, 292, 349

Spekkens, K., \& Sellwood, J. A. 2007, ApJ, 664, 204

Valenzuela, O., Rhee, G., Klypin, A., Governato, F., Stinson, G., Quinn, T., \& Wadsley, J. 2007, ApJ,657, 773

Weiner, B. J., Sellwood, J. A., \& Williams, T. B. 2001, ApJ, 546, 931

Williams, B. F., Dalcanton, J. J., Stilp, A., et al. 2010, ApJ, 709, 135

Zánmar Sánchez, R., Sellwood, J. A., Weiner, B. J., \& Williams, T. B. 2008, ApJ, 674, 797

This 2-column preprint was prepared with the AAS LATEX macros v5.2. 